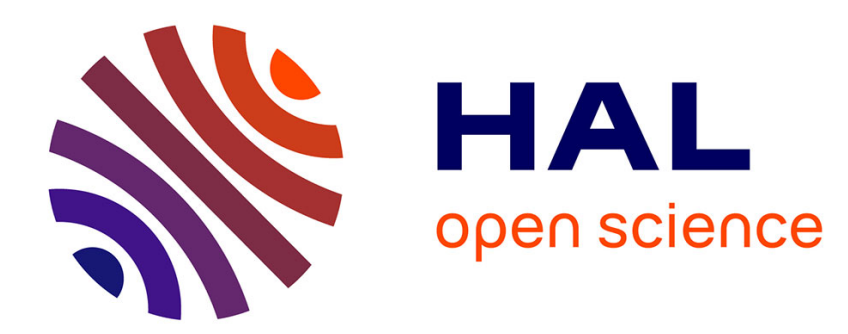

\title{
Vers une approche constructionnelle des structures au conditionnel
}

\author{
Patrick Caudal
}

\section{To cite this version:}

Patrick Caudal. Vers une approche constructionnelle des structures au conditionnel. Langue française, 2019, 200, pp.49-61. hal-01973327

\section{HAL Id: hal-01973327 \\ https://hal.science/hal-01973327}

Submitted on 8 Jan 2019

HAL is a multi-disciplinary open access archive for the deposit and dissemination of scientific research documents, whether they are published or not. The documents may come from teaching and research institutions in France or abroad, or from public or private research centers.
L'archive ouverte pluridisciplinaire HAL, est destinée au dépôt et à la diffusion de documents scientifiques de niveau recherche, publiés ou non, émanant des établissements d'enseignement et de recherche français ou étrangers, des laboratoires publics ou privés. 
“04_Caudal_DEF4”(Col. : RevueLangueFrançaise) — 2019/1/7 — 11:44 — page 1 — \#1

Patrick Caudal

Laboratoire de linguistique formelle (CNRS UMR 7110) \& Université Paris-Diderot

\section{Vers une approche constructionnelle des structures au conditionnel}

\section{INTRODUCTION}

Si un premier projet constructionnel d'analyse du conditionnel (esquissé dans Patard 2017) consistait à traiter trois "emplois » différents comme des sens homonymiques ${ }^{1}$ (et donc à postuler l'existence de morphèmes / ке/ homonymes), le travail entrepris ici s'en distinguera, ainsi que d'autres (rares) travaux constructionnels sur les «emplois" des flexions verbales (entre autres Michaelis 2011) ${ }^{2}$. Ils analysent au moins certains emplois de temps verbaux comme des constructions séparées, traitées en termes d'homonymie morphémique. Je proposerai en effet que le locus de la conventionnalisation d'au moins plusieurs " emplois » du conditionnel est plus complexe. Plutôt qu'à un ou des morphèmes / $\mathrm{\varepsilon} \varepsilon /$, les contenus sémantiques des «emplois » en question sont conventionnellement (et non productivement) appariés à des structures syntaxiques (constructions) différentes au conditionnel. Le conditionnel y apparaît comme un marqueur flexionnel contraint, sans contribution sémantique compositionnelle ni rôle productif sémantico-pragmatique. L'étude des « emplois du conditionnel » ne saurait alors se limiter à une étude des relations sémanticopragmatiques les liant (s'agit-il de polysémie/monosémie ou d'homonymie ?) au niveau du morphème, mais implique en plus de poser la question de la variabilité possible des formes portant ces interprétations (morphèmes ou constructions plus complexes ?) ; bref, d'envisager le problème dans les termes d'une théorie

1. Chez Patard, «polysémie » désigne ce qui est, pour moi, une forme d'homonymie, i.e. de sens établis comme sémantiquement autonomes/conventionnalisés, en partie encodés en mémoire. Et ses « effets de sens » correspondent à ma notion de « polysémie calculatoire ».

2. Voir Caudal (2017) pour un exemple d'analyse de ce type des « imparfaits atténuatifs ». 


\section{Du conditionnel}

des appariements formes/sens (et de l'homonymie/polysémie/synonymie en sus, si une même forme peut avoir plusieurs sens) qui puisse se déployer à divers niveaux morphosyntaxiques. Mon objectif n'est pas de proposer une solution exclusivement basée sur des constructions complexes mais mieux équilibrée et plus diversifiée dans les mécanismes invoqués. Comme A. Patard (2017), je $\mathrm{n}^{\prime}$ exclus en rien que sémantique et pragmatique interviennent pour expliquer certaines variations ; j'entends cependant attribuer aux constructions une part plus large, et par là plus juste, dans l'analyse du conditionnel, que celle plus réduite, voire minime, qu'elles ont reçue dans les approches existantes.

Mon travail s'opposera essentiellement à l'hypothèse "monosémie/polysémie forte", qui défend l'idée que tous, ou presque tous, les « emplois » majeurs du conditionnel (i.e. en dehors peut-être d'une poignée de constructions marginales) peuvent être réduits à une association entre ce morphème /вє/ et un sens unique, ou un ensemble de sens en relation de polysémie calculatoire.

De nombreux travaux revendiquent une approche monosémique/polysémique forte du conditionnel, même s'ils se concentrent sur un ou deux « emplois »; en proposer une revue détaillée est impossible dans ce format d'article. Je renvoie à A. Patard (2017) pour une critique organisée. Parmi cette abondante littérature, je relèverai une récurrence analytique importante : le rôle de la temporalité ou son absence de rôle. Par exemple dans C. Vetters (2001) ou A. Lauze (2008), le COND (conditionnel) est atemporel ou temporellement sous-spécifié tandis que chez S. Azzopardi et J. Bres $(2011,2014)$ et J. Bres (2012), il encode un composant de sens passé. Une analyse semblable prévaut chez L. Gosselin (1999), P. Caudal et C. Vetters (2005) ou encore P. Caudal (2011), le COND y étant une forme temporo-modale combinant des «morphèmes" / / / (modal) et / $\varepsilon /$ (temporel). En somme, le composant passé du COND est donné pour « vrai», «faux» / sous-spécifié (Iatridou 2000), ou absent. C'est sur ce terrain sémantique que je construirai une première critique de la thèse monosémique/polysémique forte (§ 2), comme chez A. Patard (2017). Je proposerai enfin (§ 3) un argumentaire constructionnel qui divergera, lui, d'A. Patard (2017) et portera sur les appariements formes/sens entre structures complexes au conditionnel et interprétations hypothétiques et atténuatives.

\section{ARGUMENTS SÉMANTIQUES CONTRE LA MONOSÉMIE/POLYSÉMIE CALCULATOIRE}

\subsection{Une question théorique et formelle}

Ma critique sémantique de la monosémie/polysémie calculatoire sera fondée en partie sur des considérations analytiques qui ne peuvent réellement apparaître sans traitement formel ; je vais donc esquisser une implémentation formelle des structures conditionnelles hypothétiques ( $\mathrm{SCH}$ ), qui offriront un ensemble 
de données cruciales à ma réflexion. Mon analyse sera basée sur le traitement de la modalité (v. Ramchand 2014, 2018), que je combinerai avec l'approche de P. Schlenker (2004), pour modéliser les SCH. Le schéma sous (1) définit les modaux comme des descriptions de situations ${ }^{3}$ qui, au lieu de dénoter une quantification sur des mondes possibles (Kratzer 1991), dénotent des fonctions de choix $(\mathrm{CHOIX})^{4}$ sur un ensemble de situations alternatives actives, correspondant aux possibilités accessibles par rapport au contexte $c$; l'argument $x$ est le siège (holder) du choix, déterminé par la syntaxe et/ou le contexte (ce peut être l'énonciateur, l'agent/sujet de P, etc.).

(1) $\llbracket$ Modal $\rrbracket^{g, c}=\lambda P \lambda x \exists s_{1}\left[P\left(s_{1}\right) \& s_{1}\right.$ est un CHOIX pour $x$ en $\left.c\right]$, où $s_{1} \in \operatorname{ALT}\left(s_{1}\right)$, où $\operatorname{ALT}\left(\mathrm{s}_{1}\right)=\left\{\mathrm{s}: \mathrm{s}\right.$ est une alternative active de $\left.\mathrm{s}_{1}\right\}$

De façon analogue, P. Schlenker (2004) traite les protases des structures conditionnelles comme une fonction de choix entre des alternatives (où $\mathrm{t}_{\mathrm{w} 0} \mathrm{~W}_{\mathrm{i}}$ indique que $\imath$ sélectionne les individus-mondes $w_{i}$ les plus saillants/pertinents/proches de $\mathrm{w}_{0}$ dans le contexte - i.e. l'ordre des mondes possibles est linéarisable par rapport à une relation de ressemblance), plus un quantifieur (noté par les parenthèses carrées [ ] ; la protase offre le contenu référentiel du restricteur du quantifieur qui la lie à l'apodose) :

(2) a If John came, Mary would be happy.

b. $\left[\mathrm{i}_{\mathrm{w} 0} \mathrm{w}_{\mathrm{i}} \operatorname{came}\left(\mathrm{J}, \mathrm{t}_{0}, \mathrm{w}_{\mathrm{i}}\right)\right]$ happy $\left(\mathrm{M}, \mathrm{t}_{0}, \mathrm{w}_{\mathrm{i}}\right)$

L'analyse de P. Schlenker permet de saisir une analogie entre descriptions définies et structures conditionnelles hypothétiques $(\mathrm{SCH})$, à savoir qu'elles introduisent un topique discursif (cf. l), non asserté. Combinons à présent ces deux approches en (3) pour modéliser les $\mathrm{SCH}$, en posant que leur dénotation générale implique :

- qu'est effectuée une double description de situations par deux sortes de fonctions de choix ancrées par rapport à la situation perspectivale $\mathrm{s}_{0}:$ la fonction de description définie $\imath_{s 1}$ (non assertive) présuppose l'antécédent (modal) $\mathrm{s}_{1}$ tandis que $\exists \mathrm{s}_{2}$ CHOIX $(\mathrm{x})\left(\mathrm{s}_{0}\right)\left(\mathrm{s}_{2}\right)$ quantifie existentiellement et décrit le conséquent $\mathrm{s}_{2}$ - les situations alternatives idoines étant construites par ALT ; $\mathrm{s}_{1}$ et $\mathrm{s}_{2}$ sont actives relativement à $\mathrm{s}_{0}$ - et REL est une relation d'ordre temporel ancrant $\mathrm{s}_{0}$ par rapport à l'intervalle de l'énonciation (now) ;

- qu'une relation inférentielle modale (MOD-INF) lie $s_{1}$ et $s_{2}$, telle que $s_{1}$ est une précondition de $s_{2}$ (MOD-INF est une relation inférentielle sensible au contexte discursif, distincte de l'implication logique classique ; je laisse sa définition précise ouverte).

\footnotetext{
3. V. Kratzer (2017). Les situations sont des treillis dont le plus petit élément serait un événement et le plus grand un monde possible ; elles ont des propriétés de localisation spatiotemporelle.

4. Dont la nature peut varier selon les bases modales considérées, mais je ne peux ici en dire plus.
} 


\section{Du conditionnel}

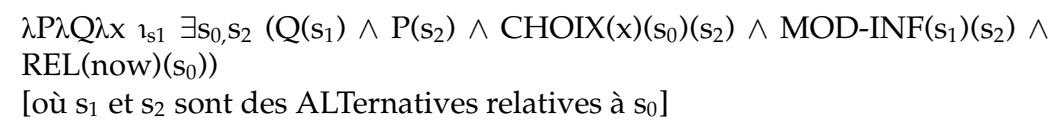

Grâce à $\mathrm{s}_{0}$, il est aisé de contraindre temporellement la perspective adoptée sur une $\mathrm{SCH}$, soit productivement, soit par un ancrage conventionnalisé dans un tour. Les relations temporelles exactes entre $s_{1}$ et $s_{2}$ sont contextuellement calculées et ne seront pas évoquées.

Je considérerai que le français présente trois types sémantiques de $\mathrm{SCH}$ pertinents pour le problème étudié ${ }^{5}$, à savoir :

- les SCH de sens contrefactuel présent ${ }^{6}$ vivace (CfPRv) $(s i+P-P R, Q-F U T)$ d'où le conditionnel est exclu ;

- les SCH contrefactuelles présentes « moins vivace » (CfPRmv) (si +P-IMPF, $Q-C O N D$ ) où $\mathrm{s}_{1}$ et $\mathrm{s}_{2}$ sont des alternatives actives en $\mathrm{s}_{0}$, au moins dans le futur, même si elles sont improbables et, de fait, inaccessibles depuis la localisation temporelle actuelle de $\mathrm{s}_{0}$;

- et les SCH contrefactuelles passées (CfPA) (si + P-PQP, Q-COND.PA) ni $\mathrm{s}_{1} \mathrm{ni}$ $\mathrm{s}_{2}$ ne sont des possibilités actives en $\mathrm{s}_{0}$; elles peuvent dans certains contextes avoir été accessibles depuis une situation antérieure à $\mathrm{s}_{0}$ (en cas de backtracking ; Ramchand 2018) mais cela n'est pas garanti a priori.

La situation perspectivale des trois types peut être présente (toutes les trois peuvent être compatibles avec des adverbiaux présents si leurs verbes sont statifs et les contextes appropriés, cf. (4)). Inversement, seules les SCH de sens CfPA sont compatibles avec des adverbiaux cadratifs de sens passé.

(4) S'il \{est / était / avait été\} malade aujourd'hui, ce \{sera / serait / aurait été\} la grippe. $^{7}$

(5) S'il avait été malade l'an dernier, ça aurait été la grippe.

(6) *S'il \{est/était\} malade l'an dernier, ce \{sera/serait $\}$ la grippe.

Les SCH CfPA sont aussi le seul type qui implique ${ }^{8}$ la fausseté des contenus propositionnels $\mathrm{P}$ et $\mathrm{Q}$, relativement au contexte contemporain de la situation de perspective. Je qualifierai donc les structures de sens CfPRv et CfPRmv de contrefactuelles ouvertes (CFO) - car elles ont des alternatives actives dans la situation d'énonciation - et les opposerai aux contrefactuelles CfPA, qui sont

5. J'exclus bien sûr de mon analyse les « indicative conditionals » au sens de Stalnaker (1975).

6. L'ancrage temporel correspondant est en fait (i) co-temporel de la situation $\mathrm{s}_{0}$ et (ii) incompatible avec des cadres temporels passés tels qu'hier. J'ignorerai ici les effets liés à du discours indirect, par exemple.

7. Ces exemples sont fabriqués pour former paradigme, mais l'on peut trouver des attestations similaires, cf. p. ex. « Dans mon esprit, cela ne signifie nullement que Foucault aurait dit la même chose s'il avait été ici aujourd'hui. » (Becker, Ewald F. \& Harcourt B. E. (2014), « Gary Becker dialogue avec Michel Foucault », Socio 3, 265-288).

8. La seule exception à cette observation sont les $\mathrm{SCH}$ au COND.PA « résultatif », cf. : Si je procrastinais moins, j’aurais déjà fini mon article. 
elles des contrefactuelles fermées (CFF) - car elles n'ont pas d'alternatives actives dans la situation de l'énonciation (tout au plus peuvent-elles parfois en avoir eu depuis une $\mathrm{s}_{0}$ passée).

\subsection{L'argumentaire sémantique}

Utilisons à présent cet appareillage théorique pour construire un argumentaire sémantique contre les analyses monosémistes/polysémistes fortes. Du fait que le COND français est issu de la morphologisation du tour latin cantare habebam et qu'il avait initialement une interprétation de type déontique passée (Coleman 1971 ; Fleischman 1982 ; Bourova 2007 ; Bourova \& Tasmowski 2007), je déduirai que son étymon avait un ancrage perspectivale passé, contrairement aux structures CfPRmv marquées au conditionnel, qui depuis le vieux français, ont un ancrage présent. Un changement sémantique est donc intervenu dans l'intervalle.

Par contraste, cependant, les « emplois temporels » du conditionnel, comme cantare habebam, sont au contraire demeurés liés à un ancrage passé, cf. (7) :

(7) Il disait autrefois qu'il deviendrait riche.

S'ajoute bien sûr à cela le fait que le « conditionnel objectif futur » (Bres 2012) est porteur d'un sens qui manifestement n'a rien de potentiel ou contrefactuel : il désigne un événement certain, i.e. ne fait intervenir aucun dispositif modal relativisant la véridicibilité de son contenu propositionnel, et implique une base modale « historique " (Portner 2009). On ne peut dériver cette différence de façon calculatoire ; elle doit être encodée - ce problème a été signalé maintes fois à propos des bases modales (v. Rullmann, Matthewson \& Davis 2008).

COND.PA et conditionnelles de sens CfPA offrent des données parallèles. $\mathrm{Si}$ en français moderne, le conditionnel passé est nettement spécialisé ${ }^{9}$ dans les configurations CfPA, qui peuvent avoir un ancrage temporel passé, avant le moyen-français, il semble que ces formes aient plutôt exprimé des sens CfPRmv résultatifs, à ancrage temporel présent (Patard, Grabar \& De Mulder 2015), cf. (8). Ce changement de prédominance sémantique suggère une collocation dans l'appariement forme/sens observé, et non un pur fait productif.

(8) [...] sel pooit savoir ne antendre que eüsses cestui amé, toz tens t'avroit mes an vilté. (Enéas, $\mathrm{XII}^{\mathrm{e}}$ )

'si celui-ci pouvait apprendre ou comprendre que tu as aimé celui-là, il te tiendrait en mépris pour toujours.'

9. Les conditionnelles au COND.PA « résultatif » sont toujours possibles en français moderne mais apparemment marginales (d'après les décomptes de Patard, Grabar \& De Mulder 2015) et sont facilitées par les modifieurs orientant vers une pertinence présente (p. ex. déjà). 


\section{Du conditionnel}

Ajoutons une dernière pièce à ce puzzle : les « emplois évidentiels inférentiels » (non reportatifs) du conditionnel ont également changé leur ancrage temporel par rapport à leurs premières attestations ; cf. (9), emprunté à V. Bourova et $P$. Dendale (2013), qui se traduit par un COND.PA en français moderne :

(9) «He, dieux», fet-il, « dont venez vous? Vos armes vendriez les vous? Ge croi, vous les avez prestees... ! » (Li romans de Claris et Laris, v. 24239, circa 1270)

'[...] D'où venez-vous donc, fait-il ? Auriez-vous vendu vos armes ? Je crois que vous les avez prêtées [...]'

Cet ancrage perspectival passé les rapproche des « emplois temporels » de l'époque plutôt que des « emplois contrefactuels » des structures au COND.PR. Un tel sens évidentiel inférentiel - mais aussi reportatif - étant, en outre, modélisable selon un type d'inférence ayant une base modale différente de celle d'une prédiction temporelle subjective ou objective (v. Vatrican 2010), une approche polysémique calculatoire semble résolument délicate à leur appliquer.

Clôturons notre exposé sémantique. Pour importants qu'ils soient, ces arguments contre la monosémie/polysémie calculatoire (ils excluent la version forte de ces hypothèses, ce qui était mon but) ont un goût d'inachevé. S'ils indiquent que des sens non calculés doivent être associés à des structures au conditionnel, ils ne permettent pas de savoir à quel niveau morpho-syntaxique les appariement forme/sens conventionnalisés interviennent. A. Patard (2017) suggère que c'est au niveau du morphème /ьع/, mais je vais à présent essayer d'identifier quelques-unes des difficultés que rencontre une telle analyse, sur un plan diachronique, synchronique et variationniste.

\section{ARGUMENTAIRE À L'INTERFACE SYNTAXE/SÉMANTIQUE}

Mon argumentaire constructionnel s'articulera autour d'un échantillon ${ }^{10}$ de données pertinentes couvrant les « emplois hypothétiques » et « atténuatifs » du COND. Il visera à révéler la complexité des paramètres morphosyntaxiques conventionnels gouvernant le marquage des structures correspondantes au conditionnel. Mon analyse sera adossée à une idée qui n'a en fait rien de nouveau : il s'agit de considérer que telle ou telle flexion dans tel ou tel type de structure morphosyntaxique peut s'y trouver conventionnalisée - i.e. sans que sa présence puisse être motivée sur un plan interprétatif purement calculatoire, comme cela est avancé dans P. Anand et V. Hacquard (2010) à propos du marquage imperfectif passé des $\mathrm{SCH}$ dans les langues romanes. Je vais ici proposer une analyse comparable pour le conditionnel dans les $\mathrm{SCH}$ et l'inscrire dans une perspective théorique et formelle qualifiable de « constructionnelle ». Il ne

10. Une étude de corpus étendue devrait lui servir de fondement ; je dois ici remettre une telle étude à plus tard. 
s'agira pas uniquement d'étudier les conditionnels présent et passé, mais le système de marquage flexionnel multiple conventionnalisé qu'ils forment par alternance et combinaison - ou absence d'alternance et de combinaison - y compris avec d'autres flexions verbales, dont l'imparfait, le plus-que-parfait, le futur et les subjonctifs imparfait et plus-que-parfait.

\subsection{Structures bi-propositionnelles : " emplois hypothétiques " et SCH}

De multiples types de $\mathrm{SCH}$ bi-propositionnelles existent en français. Le plus largement étudié ${ }^{11}$ est sans doute le type si $\phi, \psi$. Les marquages possibles y sont multiples mais aussi contraints, cf. Tableau 1.

Tableau 1 : Marquage flexionnel des SCH en si $\phi, \psi$ en français moderne normatif

\begin{tabular}{|l|l|}
\hline Protase & Apodose \\
\hline IMPF & COND.PR /SUBJ.IMPF/COND.PA-résultatif \\
\hline PQP & COND.PA/SUBJ.PQP \\
\hline SUBJ.IMPF & COND.PR/SUBJ.IMPF \\
\hline SUBJ.PQP & COND.PA/SUBJ.PQP \\
\hline
\end{tabular}

Le fait saillant, et connu, est l'impossibilité de faire figurer le COND.PR ou le COND.PA dans la protase de ces structures. Pourtant, dans une large période s'étendant du moyen français au français classique, le COND pouvait y apparaître (LeBlanc 1999) ; et il le peut aussi en français populaire moderne et dans de nombreuses variétés d'Amérique, cf. (10) :

(10) Si je pourrais aller le voir, j'irais le voir. (français d'Ottawa-Hull ; LeBlanc, 1999:36)

Or, l'inventaire sémantique des « emplois » du COND en français classique, et à plus forte raison dans les variétés non-normatives, n'est pas foncièrement différent de celui du français moderne normatif ; une approche monosémique/polysémique est donc impuissante à expliquer cette variation (Caudal \& Roussarie 2005). Il faut l'attribuer à une conventionnalisation de la distribution du COND - et de l'imparfait - dans ces structures.

Par contraste, le COND peut marquer les protases de très nombreuses autres $\mathrm{SCH}$ bi-propositionnelles, même dans les variétés normatives modernes. C'est par exemple le cas dans les constructions hypothétiques en «et à subordination gauche » (au sens de Culicover \& Jackendoff 1997) (11) - ou des tours en apparence parataxiques, sans $\mathrm{et}$, de sens comparable (12). Ils partagent une intonation contrainte ; aucun contour descendant (de type « fin de déclarative ») ne peut

11. Faute de place, je passerai ici sur les structures SCH à protase réduite (p. ex. un mètre de plus/sans $S N$, COND...). 


\section{Du conditionnel}

s'appliquer à la première proposition. Ce ne sont donc pas des phrases complexes conjonctives (Corminboeuf 2009), mais des constructions bi-propositionnelles.

(11) Tu aurais refusé, et il t'aurait licencié.

(12) Vous m'offririez des fleurs, je les refuserais! (Montherlant, Celles qu'on prend dans ses bras, 1950)

Cela signifie que, même indépendamment de la diachronie et des variétés non normatives, la distribution du COND dans les protases ne peut s'expliquer sur une pure base sémantique en français moderne : si l'on suppose que (11) et (12) dénotent le type de forme logique proposé en (3) pour les SCH en si $\phi$, $\psi$ (et cela me semble raisonnable, au-delà de possibles différences sémantiques fines), on voit mal comment le COND pourrait marquer la position correspondant au préjacent de MOD-INF dans une structure, et pas dans l'autre. Le problème s'aggrave encore si l'on considère la possibilité d'employer l'imparfait en combinaison avec le COND-PA (mais pas le COND.PR) dans ces structures, cf. (13) :

(13) Tu appuyais sur ce bouton, et la bombe aurait explosé.

Il est bien connu que le conditionnel ne s'est que progressivement multiplié dans des systèmes de constructions hypothétiques bi-propositionnelles (Wagner 1939 ; Harris 1986), notamment de type si A, B. Si le COND.PR semble avoir tôt dominé dans les $\mathrm{SCH}$ de sens CfPRmv, le COND.PA, en revanche, ne s'est généralisé dans les $\mathrm{SCH}$ de sens CfPA que tardivement (cf. 2.2). Auparavant, les subjonctifs imparfait et plus-que-parfait abondaient dans les $\mathrm{SCH}$ de sens CfPA. Issu d'un ancien paradigme de plus-que-parfait indicatif latin, le subjonctif imparfait a en effet longtemps conservé la possibilité de marqueur des $\mathrm{SCH}$ de sens CfPA dans les SCH, cf. (14) :

(14) Sem(e) creïsez, venuz i fust mi sire.

'Si vous m'aviez cru, mon roi serait venu.' (Roland, 1728)

Je vois dans ce phénomène complexe la clé du mystère de l'émergence du marquage imparfait des $\mathrm{SCH}$ à sens CfPRmv : associé au plus-que-parfait indicatif (l'ancêtre du SUBJ.IMPF) dans des tours CfPA en latin tardif ou en galloroman, l'imparfait aurait suivi par solidarité constructionnelle, la " glissade » ${ }^{12}$ progressive de ce dernier, du statut de marque de tours CfPA à celui de marque de tours CfPRmv - et, de fait, en français contemporain, le SUBJ.IMPF ne marque plus les structures de sens CfPA. L'éviction ultérieure quasi complète du SUBJ par le COND dans ces tours a fait le reste ${ }^{13}$.

S'il n'est pas indispensable de poursuivre notre inventaire des $\mathrm{SCH}$ bipropositionnelles, on doit néanmoins noter que ces phénomènes de distribution

12. Cette « glissade » par tuilage sémantique $\{C f P A\}>\{C f P A ; C f P R m v\}>\{C f P R m v\}$ peut se modéliser à l'aide des notions de bridging et de switch context de Heine (2002).

13. Dans un travail de plus grande ampleur, il faudrait aussi évoquer le rôle de la diachronie de l'analogie morphologique entre COND.PR et imparfait. 
contrainte sont extrêmement abondants - p. ex. avec que relateur propositionnel, avec ou sans protase interrogative polaire, etc., cf. (15)-(16) :

(15) Eussent-ils choisi de négocier leur installation, les Anglais auraient donc eu fort à faire. (Préaud 2010)

(16) Il serait malade que cela ne m'étonnerait guère. (Le Trait d'Union, $\mathrm{n}^{\circ} 45,1957$ )

On relèvera l'impossibilité d'employer l'imparfait et le plus-que-parfait dans ces structures, seuls ou en combinaison avec le COND, par opposition à (13). De même, aucune variante CfPRv n'est possible avec le subjonctif présent et/ou le futur, contrairement aux $\mathrm{SCH}$ en si $\phi, \psi$ pour le futur. On pourrait multiplier presque à l'infini les contraintes flexionnelles difficiles à expliquer sur une pure base sémantique.

Pour résumer, ces différents faits convergent pour démontrer que les combinaisons flexionnelles trouvées dans les différents types de structures $\mathrm{SCH}$ sont de nature conventionnelle. Le conditionnel serait alors vu comme pris dans un faisceau de conventionnalisations le dépassant ; il n'est que l'un des multiples ingrédients conventionnels (intonation, syntaxe positionnelle et constructionnelle, autres marqueurs flexionnels co-distribués dans la structure, etc.) de ces structures. Avec les tours bi-propositionnels, la question des combinaisons avec une autre flexion sur les deux positions syntaxiques à marquer, ou des alternances avec d'autres flexions sur une même position syntaxique, est aussi à expliquer. On ne saurait traiter un tel phénomène comme un cas d'homonymie morphémique : les appariements sens/formes concernent des structures beaucoup plus complexes que des morphèmes.

\subsection{Structures mono-propositionnelles : "emplois atténuatifs " et tours modaux, péri-modaux et interactionnels}

Passons à présent aux «emplois atténuatifs » du COND. Ils ont un ancrage perspectival présent au COND.PR, au sens où ils refusent les cadres temporels passés (17), ce qui les rapproche des « emplois hypothétiques ». Inversement, leur ancrage peut être passé au COND.PA - j'y reviendrai infra.

\{Il faudrait / Vous devriez / Je voudrais\} partir ( ${ }^{*}$ hier).

Mais de façon remarquable, il apparaît que certaines constructions modales épistémiques pourtant capables «d'emplois atténuatifs » au COND.PR sont incompatibles avec le COND.PA (18) :

(18) Il se pourrait ( ${ }^{*} \mathrm{~s}^{\prime}$ aurait pu) que l'on doive retarder les élections. (Libération, 18-05-2011)

Si cela suffit à prouver que certains tours modaux ont une interaction conventionnalisée, et non calculatoire, avec le conditionnel, il convient néanmoins d'élargir notre enquête. 


\section{Du conditionnel}

Les « emplois atténuatifs » sont, de fait, essentiellement limités à certains verbes de communication et $\mathrm{d}^{\prime}$ argumentation à forte dimension interactionnelle (p. ex. dire, objecter, faire remarquer), aux verbes et constructions modales (surtout de sens dynamique, donc interactionnel, cf. vouloir, devoir, pouvoir, avoir besoin de, falloir, avoir quelque chose / un service à PRO demander...) et à certaines combinaisons contraintes des deux (vouloir dire, vouloir savoir...). Ils s'étendent aussi aux tours péri-volitionnels basés sur des constructions évaluatives (préférer, être préférable, convenir (mieux)...) et aux expressions évaluatives et attitudinales employées dans des interactions sociales feutrées (cf. être plutôt d'avis de/que, avoir (plutôt) le sentiment que...). Ces limitations suggèrent, selon moi, qu'il s'agit d'un réseau constructionnel de tours conventionnalisés, mais une étude détaillée reste à mener ${ }^{14}$. Avec les modaux épistémiques marqués au COND, les constructions obtenues ont un sens très difficile à distinguer des emplois " évidentiels » : l'énonciateur atténue la prise en charge du jugement épistémique (d'où des effets contextuels semblables, parfois).

Le caractère conventionnalisé, et en fait, lexifié, de beaucoup de ces tours devient apparent dès lors que l'on s'intéresse à la variation de leurs interprétations et à la distribution des formes COND.PA et COND.PR. Le point le plus remarquable tient à l'émergence, apparemment récente, d'une lecture " ultraatténuative » à ancrage perspectival présent (co-temporel de la situation d'énonciation, disons) des tours modaux et péri-modaux au COND.PA. (19) a ainsi un ancrage perspectival présent et une valeur atténuative marquée :

(19) - Bonsoir, Monsieur, j'aurais voulu vous voir...

- Ah... Ce soir, ça va être un peu juste. C'est urgent ? (Winckler, La maladie de Sachs, 1998)

L'émergence de cette lecture $\mathrm{d}^{\prime}$ « ultra-atténuation » était rendue nécessaire, à mon avis, par le caractère très conventionnalisé en français moderne de vouloirCOND.PR, qui le rendait de moins en moins clairement « atténuatif » dans un cycle évolutif liée à une érosion sémantique (de nouvelles formes marquées venant remplacer celles érodées) qui rappelle, par exemple, celui de la négation multiple. On remarquera que ces lectures ultra-atténuatives n'apparaissent pas à la même époque pour tous les verbes/tours; alors que vouloir présente (à ma connaissance) sa première occurrence en 1788 dans Frantext (20), désirer n'en offre aucune avant 1857 (21) :

(20) Mme DE ROSELLE. - Mais, entre nous, pourtant, j'aurois voulu savoir... M. DE PLINVILLE. - Savoir ? quoi ?

(Collin D'Harleville, L'Optimiste ou l'Homme toujours content, 1788)

14. Fait frappant, ce réseau constructionnel recouvre en partie celui de l' «imparfait atténuatif »: leurs différences révèlent la nature conventionnalisée de ces marquages flexionnels en même temps qu'une base sémantique convergente dans les recrutements faits, relevant d'un effet d' « analogisation » / « gang effect » (Bybee 2001). 
“04_Caudal_DEF4”(Col. : RevueLangueFrançaise) — 2019/1/7 — 11:44 — page 11 — \#11

Vers une approche constructionnelle des structures au conditionnel

(21) (À Edouard Houssaye). Décembre 1856 ou Janvier 1857. Mon cher ami, Je vous ai apporté les épreuves, j'aurais désiré que Théo les lût. Il y a une phrase peut-être indécente ??? (Flaubert, Correspondance, 1854-1857)

Par contraste, la périphrase volitionnelle avoir envie n'a aucune occurrence de ce type, ni dans Frantext ni dans $\mathrm{FrWaC}$ (en dépit d'un nombre d'occurrences au COND.PA comparable à celui de désirer ou souhaiter). Google permet de trouver des attestations peu normatives en forum de discussion, ce qui suggère un changement en cours. Enfin, hors des tours et verbes modaux, l'emploi ultraatténuatif semble impossible :

(22) Il vous a rencontré en 1930 rue de la Pompe... Je dirais (*j'aurais dit) : 1928 ou 1929. (Mauriac, Le Temps Accompli, 1991)

Toutes ces données suggèrent que les emplois « atténuatifs » du conditionnel renvoient en réalité à un réseau complexe de constructions modales, périmodales ou interactionnelles, dont les interprétations sont fixées par conventionnalisation, et non calculées, et qui semble encore en pleine évolution.

\section{POUR CONCLURE}

La conclusion de cette étude programmatique est bien entendu partielle et en devenir. La section 2 a établi que, sur un plan sémantique, il était douteux que l'on puisse proposer une analyse monosémique/polysémique forte de tous les «emplois» du conditionnel. Il a ensuite été avancé (§3) qu'une approche constructionnelle basée sur l'hypothèse de morphèmes conditionnels homonymiques (Patard 2017) était insuffisante. Dans au moins deux types $\mathrm{d}^{\prime}$ « emplois du conditionnel » (atténuatifs et hypothétiques), la distribution des marquages flexionnels ne paraît pas explicable sans supposer qu'ils impliquent un appariement sens/formes conventionnalisé directement au niveau des constructions complexes (structures bi-propositionnelles hypothétiques ou structures modales/péri-modales/ interactionnelles atténuatives). D'autres types d' « emplois » - évidentiels et temporels, notamment - auraient pu et dû être étudiés à cet égard. On trouvera dans A. Patard (2017) de nombreuses observations diachroniques, qui suggèrent que ces derniers ont autrefois subi des limitations ou collocation syntaxiques, qui sont de forts indices de conventionnalisation des emplois au niveau de constructions complexes. Il semble, en revanche, que ces contraintes de forme aient au moins en partie disparu en français moderne, ce qui accréditerait l'idée que ces « emplois » du conditionnel (ou au moins certains) impliquent bel et bien des " morphèmes homonymes ", comme proposé par A. Patard (2017). Mais cela restera une hypothèse à évaluer au futur... sinon au conditionnel. 
“04_Caudal_DEF4” (Col. : RevueLangueFrançaise) — 2019/1/7 — 11:44 — page 12 — \#12

Du conditionnel

\section{Références bibliographiques}

ANAND P. \& HACQUARD V. (2010), "The role of the imperfect in Romance counterfactuals", Proceedings of Sinn und Bedeutung, vol. 14, 37-50.

AZZopARDI S. \& BrES J. (2011), "Temps verbal et énonciation. Le conditionnel et le futur en français : I'un est dialogique, l'autre pas (souvent) ", Cahiers de praxématique 56, 53-76.

AZZOPARDI S. \& BRES J. (2014), " Futur, conditionnel, et effets de sens de conjecture et de rejet en interrogation partielle ", dans F. Neveu et alii (éds), 4e Congrès Mondial de Linguistique Française - CMLF 2014, Berlin (Allemagne), 3003-3013.

BOUROVA V. (2007), "Les constructions latines Infinitif+habebam vs Infinitif+habui et le développement du conditionnel roman ", dans D. Trotter (éd.), Actes du XXIV Congrès International de Linguistique et de Philologie Romanes, vol. 2, Tübingen, Max Niemeyer, 461-474.

BOURova V. \& DendaLE P. (2013), "Serait-ce un conditionnel de conjecture ? Datation, évolution et mise en relation des deux conditionnels à valeur évidentielle ", Cahiers Chronos 26, 183-200.

BOUROVA V. \& TASMOWSKI L. (2007), "La préhistoire des futurs romans : ordre des constituants et sémantique ", Cahiers Chronos 19, 25-41.

BRES J. (2012), "Conditionnel et ultériorité dans le PASSÉ : de la subjectivité à l'objectivité ", dans F. Neveu et alii (éds), $3^{e}$ Congrès Mondial de Linguistique Française - CMLF 2012, Lyon (France), 1719-1730.

ByBEe J. (2001), Phonology and Language Use, Cambridge, Cambridge University Press.

CAUDAL P. (2011), "Towards a novel aspectuo-temporal account of conditionals", Cahiers Chronos 22, 179-209.

CAUDAL P. (2017), "Les <usages atténuatifs> de l'imparfait entre conventionnalisation locale et compositionnalité globale : vers une analyse constructionnelle ", dans E. Hrubaru, E. Moline \& A. Velicu (éds), Nouveaux regards sur le sens et la référence. Hommages à Georges Kleiber, Cluj, Echinox, 179-256.

CaUdal P. \& Roussarie L. (2005), " Sémantique et pragmatique des propositions en SI ", Cahiers Chronos 12, 51-66.

CAUDAL P. \& VetTers C. (2005), " Un traitement conjoint du conditionnel, du futur et de l'imparfait : les temps comme des fonctions d'acte de langage ", Cahiers Chronos 12, 109-124.

Coleman R. (1971) "The origin and development of Latin habeo+infinitive", The Classical Quarterly 21 (1), 215-232.

CORMInBoEUf G. (2009), L'expression de l'hypothèse en français : entre hypotaxe et parataxe, Bruxelles, De Boeck.

CULICOVER P. W. \& JACKENDOFF R. (1997), “Semantic subordination despite syntactic coordination”, Linguistic Inquiry 28 (2), 195-217.

FleISCHMAN S. (1982), The Future in Thought and Language, Cambridge, Cambridge University Press.

Goldberg A. E. (1995), Constructions: A Construction Grammar Approach to Argument Structure, Chicago, University of Chicago Press.

GosseliN L. (1999), "Les valeurs de l'imparfait et du conditionnel dans les systèmes hypothétiques ", Cahiers Chronos 4, 29-51.

HARRIS M. B. (1986), “The historical development of si-clauses in Romance”, in E. Traugott et alii (eds.), On Conditionals, Cambridge, Cambridge University Press, 265-284.

HEINE B. (2002), "On the role of context in grammaticalization", in I. Wischer \& G. Diewald (eds.), New Reflections on Grammaticalization, Amsterdam, Benjamins, 83-101. 
Vers une approche constructionnelle des structures au conditionnel

IATRIDOU S. (2000), “The grammatical ingredients of counterfactuality”, Linguistic Inquiry 31 (2), 231-270.

Kratzer A. (1991), "Modality", in A. von Stechow \& D. Wunderlich (eds.), Semantics: An International Handbook of Contemporary Research, Berlin, De Gruyter, 639-650.

KRATZER A. (2017), "Situations in natural language semantics", in E. N. Zalta (ed.), The Stanford Encyclopedia of Philosophy, Stanford University.

LAUZE A. (2008), "Le conditionnel : forme temporelle ou atemporelle ? ", Communication, lettres et sciences du langage 2 (1), 92-106.

LeBlanc C. L. (1999), Du conditionnel dans les propositions hypothétiques en " si " : cet Intrus, PhD thesis, University of Ottawa (MA),

MichaeLIS L. A. (2011), “Stative by construction”, Linguistics 49 (6), 1359-1399.

PATARD A. (2017), "Du conditionnel comme constructions ou la polysémie du conditionnel ", Langue française 194, 105-124.

Patard A., Grabar N. \& De Mulder W. (2015), "Étude diachronique du conditionnel passé ou l'origine de la contrefactualité ", Journal of French Language Studies 25 (2), 189-211.

PORTNER P. (2009), Modality, New York, Oxford University Press.

PrÉAUd M. (2010), "La terre à plusieurs ", Multitudes 41, 82-90.

RAMCHAND G. C. (2014), "Stativity and present tense epistemics", Proceedings of the 24th Semantics and Linguistic Theory Conference - SALT 24, New York (USA), 102-121.

RAMCHAND G. C. (2018), Situations and Syntactic Structures: Rethinking Auxiliaries and Order in English, Cambridge (MA), MIT Press.

Rullmann H., Matthewson L. \& Davis H. (2008), “Modals as distributive indefinites”, Natural Language Semantics 16 (4), 317-357.

SCHLENKER P. (2004), "Conditionals as definite descriptions", Research on Language and Computation 2 (3), 417-462.

Stalnaker R. (1975), “Indicative conditionals”, Philosophia 5 (3), 269-286.

VATRICAN A. (2010), "La modalité et le <conditionnel de rumeur> en français et en espagnol ", Modèles linguistiques XXXI (62), 83-94.

VETTERS C. (2001), "Le conditionnel : ultérieur du non-actuel ", dans P. Dendale \& L. Tasmowski (éds), Le conditionnel en français, Metz, Université de Metz, 169-207.

WAGNER R. L. (1939), Les phrases hypothétiques commençant par " si " dans la langue française, des origines à la fin du Xvl ${ }^{e}$ siècle, Paris/Genève, Droz. 


\section{ABSTRACTS}

Patrick Caudal, Towards a constructional account of structures in the conditionnel

This paper will put forth an innovative research program on the French conditionnel, backing it with some relatively detailed empirical arguments. The research hypothesis here advanced can be described as constructionalist in the broadest sense (and not simply in the sense of Goldberg 1995). The main idea defended here is that the diversity of the so-called 'uses' (emplois) of the conditional cannot be accounted for by means of a single semantic invariant (monosemy), nor purely through productive polysemous or homonymous relations between various readings. Rather, it sometimes implies conventionnalized forms/meanings pairings at the level of syntactically complex constructions.

Keywords : conditional, modality, constructions, monosemy, polysemy

\section{RÉSUMÉS}

\section{Patrick Caudal, Vers une approche constructionnelle des structures au condition- nel}

Le présent article s'efforcera d'esquisser un programme de recherche novateur sur le conditionnel et d'avancer quelques arguments empiriques assez détaillés pour étayer l'hypothèse proposée. On peut la qualifier de " constructionnelle " (au sens très large du terme, et pas simplement de Goldberg 1995). L'idée principale qu'il défend est que la diversité des "emplois " du conditionnel ne saurait s'analyser ni seulement à l'aide d'un invariant sémantique (monosémie), ni uniquement par des interprétations en relation de polysémie productive vs d'homonymie, et qu'elle implique parfois des appariements formes/sens conventionnalisés au niveau de constructions syntaxiquement complexes.

Mots-clés : conditionnel, modalité, constructions, monosémie, polysémie 